\title{
Ambulatory Seizure Monitoring: From Concept to Prototype Device
}

\author{
Mark H. Myers ${ }^{a} \quad$ Madeline Threatt ${ }^{c} \quad$ Karsten M. Solies ${ }^{c}$ Brent M. McFerrin ${ }^{c}$ \\ Lindsey B. Hopf ${ }^{c} \quad$ J. Douglas Birdwellc Karl A. Sillay ${ }^{\mathrm{b}, \mathrm{c}}$ \\ Departments of a Anatomy and Neurobiology and beurosurgery, University of Tennessee Health Science Center, \\ Memphis, Tenn., and 'Department of Electrical Engineering and Computer Science, University of Tennessee, \\ Knoxville, Tenn., USA
}

\section{Key Words}

Brain computer interface $\cdot$ Phase lock value $\cdot$ Seizure monitoring

\begin{abstract}
Background: The brain, made up of billions of neurons and synapses, is the marvelous core of human thought, action and memory. However, if neuronal activity manifests into abnormal electrical activity across the brain, neural behavior may exhibit synchronous neural firings known as seizures. If unprovoked seizures occur repeatedly, a patient may be diagnosed with epilepsy. Purpose: The scope of this project is to develop an ambulatory seizure monitoring system that can be used away from a hospital, making it possible for the user to stay at home, and primary care personnel to monitor a patient's seizure activity in order to provide deeper analysis of the patient's condition and apply personalized intervention techniques. Methods: The ambulatory seizure monitoring device is a research device that has been developed with the objective of acquiring a portable, clean electroencephalography (EEG) signal and transmitting it wirelessly to a handheld device for processing and notification. Result: This device is comprised of 4 phases: acquisition, transmission,
\end{abstract}

processing and notification. During the acquisition stage, the EEG signal is detected using EEG electrodes; these signals are filtered and amplified before being transmitted in the second stage. The processing stage encompasses the signal processing and seizure prediction. A notification is sent to the patient and designated contacts, given an impending seizure. Each of these phases is comprised of various design components, hardware and software. The experimental findings illustrate that there may be a triggering mechanism through the phase lock value method that enables seizure prediction. Conclusion: The device addresses the need for long-term monitoring of the patient's seizure condition in order to provide the clinician a better understanding of the seizure's duration and frequency and ultimately provide the best remedy for the patient.

(c) 2016 S. Karger AG, Basel

\section{Introduction}

Seizure activity is characterized by recurrent, shortterm electrical discharges of the cerebral cortex that result in intermittent disturbances of brain function. The state between seizures, known as interictal behavior, appears

\section{KARGER}

E-Mail karger@karger.com

www.karger.com/aon
(C) 2016 S. Karger AG, Basel

0972-7531/16/0232-0100\$39.50/0
Mark H. Myers

Department of Anatomy and Neurobiology

University of Tennessee Health Science Center

Memphis, TN 38103 (USA)

E-Mail mhmyers@memphis.edu 


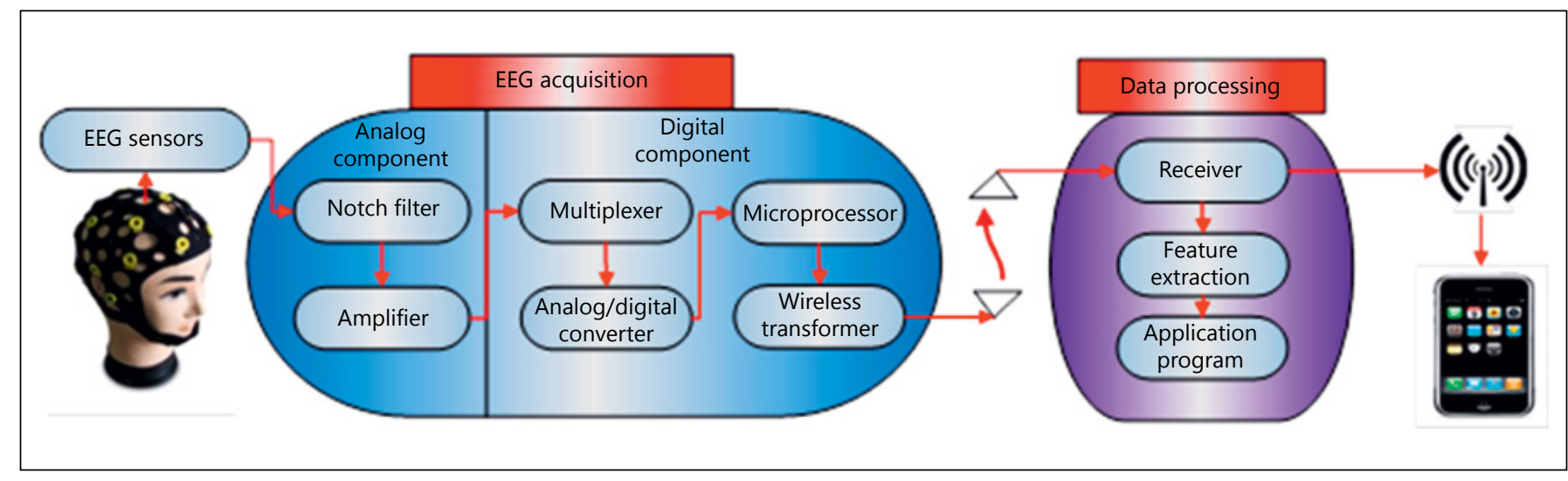

Fig. 1. Block diagram of a typical wireless BCI system. EEG data are sent wirelessly to a mobile health device (smartphone) and can be forwarded to a registry, electronic health record or other data connection.

to have minor spiking activity. In seizures of focal onset (e.g., focal seizures and partial seizures), the anatomical distribution of the interictal spikes varies, but spikes tend to occur most commonly in the epileptogenic zone and its connections. During the seizure, organized, semi-periodic electrical discharges develop in the epileptogenic zone and spread, within seconds, over widespread areas of cerebral cortex. An approach to seizure prediction and detection uses phase lock values (PLVs) and calculates the difference of phase between electroencephalography (EEG) electrodes, which are local and remote to the epileptic event. PLV is used as the seizure prediction marker and signifies the emergence of abnormal neuronal activations through local neuron populations or the epileptogenesis. The described development of an enhanced seizure detection device in this paper uses PLV to process EEG data obtained from electrodes on the scalp of an individual and wirelessly communicates to a smartphone device, which alerts a physician, emergency response system, family member or designee of an impending seizure attack. The smartphone will provide the alert mechanism by displaying and alerting the caretaker of the pending seizure attack, with vibration, audioalert or other via other designated method of the likelihood of seizure or presumed risk. This system will serve as a safety monitor that can be inquired post-event detection to obtain objective measurements that should correlate with the neurological status and health of the subject. A cap with EEG electrodes will be used for non-invasive EEG data collection with the electrodes connected to a smartphone. This device serves as a first generation prototype for development of an implantable detection system that can identify and alert to an impending seizure.

Ambulatory Seizure Monitoring: From

Concept to Prototype Device
The development of advanced EEG electrodes and signal processing technologies, which measure brain signals precisely with low noise, is the most important challenge. Practically, the signal acquisition part of general wireless brain computer interface (BCI) systems only contains a signal acquisition circuit and a microprocessor-based embedded system for transmission of the measured EEG signals. Dry electrodes have the clear advantage over electrodes that need conductive gels or glues for installation, causing depreciation of the acquired EEG signal and irritation over time. Active electrodes contain amplifier or buffer circuits integrated to the electrodes themselves [1, 2]. These amplifier or buffer circuits are located between the electrodes and the signal acquisition front end. They match scalp impedance to the requirements of the data acquisition system, providing high input impedance at the electrode-amplifier interface, while active circuits provide gain and attenuate noise. This is desired for dry electrodes that do not use conductive fluids. The low output impedance of the amplifier eliminates artifacts caused by posture changes in mobile environments, maintaining the quality of measured physiological signals.

Figure 1 features a BCI device that is composed of EEG data acquisition and data processing components. In the analog frontend stage, the amplifier and bandwidth limiter circuits are included to enable a robust and reliable acquisition of EEG signals from the sensitive raw signals. Because the amplitude of EEG signals is small compared to the measurement of EEG signals directly from the cortex, pre-amplification of the measured EEG signals at the analog frontend is extremely important. In this amplification process, wireless BCI systems typically use operational amplifiers or instrumentation amplifiers. High in- 
put impedance is necessary to match scale impedance, and it is expected that a gain on the order of $10^{3}-10^{5}$ is necessary to amplify sensed voltage levels on the order of $10 \mu \mathrm{V}$ to levels on the order of $100 \mathrm{mV}-1 \mathrm{~V}$ required by typical analog to digital converters (ADC). However, the precise amplification gain of the analog frontend will depend on the components ofthe digital system. We also need a frequency filtering procedure to remove various noise components. Seizure prediction frequencies found in EEG signals occupy a narrow bandwidth: normally from $0.1 \mathrm{~Hz}$ to less than $50 \mathrm{~Hz}$. Analog filtering will be used to remove DC signal components (low pass filter) and eliminate aliasing effects causedby the ADC (high pass filter), while digital filtering can be used more efficiently to extract useful signals from the desired frequency bands. The analog filter will also be used to attenuate power line noise, particularly noise at $120 \mathrm{~Hz}$ and its harmonics, which is caused by inductive coupling from electrical devices such as fluorescent lighting to sensor leads. These filtering processes are performed using active filtering circuits [3]. In the digital system stage, 4 integrated circuits are included: a multiplexer, an ADC, a microprocessor and a wireless transmission unit. A sigma-delta ADC can be effectively utilized to obtain a high resolution digital signal and effect high quality pass band filtering in the digital domain. Most EEG-based wireless BCI systems support multichannel recording.

Simultaneous measurement of multichannel signals requires multiple sample-and-hold circuits with low clock skew, and a multiplexer may be used to sample all of the channels using a single ADC. Given the low cost of sigmadelta ADC devices, however, it may be cost-effective to utilize multiple ADCs rather than a multiplexed solution. This tradeoff will be investigated during the design phase. The sampling frequency of the ADC is determined by the selected ADC, the speed of the microprocessor, the bandwidth available for wireless transmission and frequency range of interest in the EEG signals. The microprocessor packs the corrected EEG data into the packet buffer and hands the buffer's content to the wireless transmission unit, using an alternate buffer while the transmission takes place. The microprocessor also manages the components of the entire system. In a typical system, the microprocessor and wireless transmission units are each implemented primarily in a single integrated circuit, or package, with additional components to supply power and couple the signal to an antenna. These components can be implemented on a single circuit board module roughly the size of a pack of cards. Some wireless BCI systems load the feature extraction algorithm on the microprocessor to pro- cess the EEG signals internally $[4,5]$. Because the recorded multichannel EEG data are transmitted from the portable EEG acquisition device to the host system, the wireless transmission unit is essential. Bluetooth has many advantages such as sufficient transmission rates and wide accessibility. Thus, many wireless BCI systems employ this transmission module. Including the analog frontend and digital system stage, the acquisition unit of wireless BCI systems generally operates using onboard power sources such as Li-ion, Li-polymer and NiMH batteries. The data processing module consists of the receiving module to intercept processed EEG signals from the EEG data acquisition module, seizure feature extraction and a user interface (UI) application to alert the user of the incoming seizure. The data processing component will also transmit to the user and caregiver incoming seizure alerts so that they may be able to aid the seizure patient in managing his or her condition.

Seizure feature extraction is accomplished via a seizure prediction measurement which is implemented to determine when neurological signals across the scalp behave synchronously and align in movement. This measurement is used to determine if EEG signals will either synchronize or operate independently [6]. On the y-axis, as the measured signal moves closer to ' 1 ', the neurological signals are behaving in a synchronous manner, which is the signature behavior of seizure activity. As neurological signals synchronize, the measured signal will cross a threshold signifying that a seizure is about to occur. After a period of time, the neurological signals will appear closer to ' 1 ', signifying the brain has moved into the seizure state.

A general methodology is presented to evaluate the performance of time series analysis techniques with respect to seizure prediction. EEG data are recorded from different electrode contacts [7-9]. The mean phase coherence is determined through PLV calculations of the EEG data using a sliding window technique. The optimal threshold is determined retrospectively as a customized patient stimulation signal. For a proper prediction, the alarm must be set within a proper distance before a seizure occurrence. A threshold that is too low will result in false alarms, while a threshold that is too high will cause the device to fail to detect the onset of the seizure state. Initially, a time interval called the seizure prediction horizon SPH is chosen, such that an alarm is raised if the PLV synchrony level stays above the threshold longer than the SPH (fig. 2). When the device detects the SPH state, an alert is sent wirelessly to the patient and caregiver's smartphones to alert them of the pending seizure event [11-13]. 
Fig. 2. PLV-EEG output. Prediction marker $(\mathrm{P})$ and seizure event $(\mathrm{S})$ are signified by arrows. Prediction markers and seizure events are found by the rise of PLV values above a patient-based threshold [10].

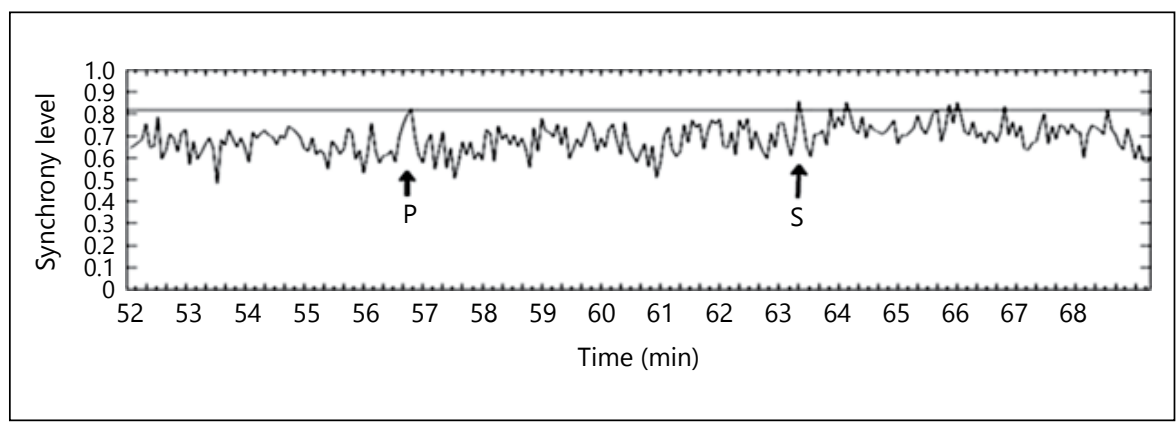

\section{Materials}

EEG data were acquired through IRB permission from the University of Memphis Institutional Review Board under exempt status, since the data were collected previously for clinical use, and there was no patient identifying markers on the data sets.

\section{Olimex EEG-AE and EEG-PE Electrodes}

Two main types of electrodes are currently available in the medical market: active and passive. Passive electrodes are less expensive; however, they require a conductive gel or glue to secure the connection to the area being sensed. Without the glue, accurate brain signals could not be acquired. Because the device requires a non-intrusive application to the patient, active electrodes were required.

The active electrodes allow brain signals to be read without a glue or adhesive being applied. This makes the device less intrusive to the user. Olimex EEG-AE and EEG-PE electrodes were chosen to acquire the EEG signals. These dry electrodes are inexpensive and open source.

\section{Olimex-SMT Amplifier}

The brain consists of billions of neurons that send electrical charges from one to another, creating electrical potential oscillations that can be measured using a voltmeter. Because these voltages are very small, a device is required to bring the amplitude of the brain signals up to a level that can be measured and interpreted. Therefore, the defining objective for an EEG-specific amplifier requires a combination of high gain and low noise operation. In this pursuit, the solutions below were considered. The open source Olimex-SMT amplifier was chosen to amplify the EEG signals acquired from the Olimex EEG-AE and EEG-PE electrodes. It is a 2-channel USB EEG amplifier that combines both the analog and digital boards of the modular EEG onto one chip. The amplifier is inexpensive and robust. Tools utilized for amplifier development involved 'EEG-SMT development board User's Manual; Revision H, April 2013', OLIMEX Ltd., 2013, 'EEG-SMT schematic' OLIMEX Ltd., 2013, 'FTDI FT232R USB UART IC' Future Technology Devices International Ltd., 2010, and 'DUAL DIGITAL ISOLATORS’ Texas Instruments Inc., 2012.

\section{Bluetooth Module}

A simple Bluetooth module, the HC-06, was chosen to transmit the amplified EEG signals. The HC-06 is configured to be a slave device that transmits the serial data from the Olimex-SMT board. The Bluetooth module was reconfigured so that the baud rate matched that of the Olimex-SMT.

Ambulatory Seizure Monitoring: From

Concept to Prototype Device

\section{Android Phone}

The mobile application was essentially the heart of the system. It has to set up and maintain all of the Bluetooth connectivity, process the data, control the patient and health provider settings and invoke the notification system. Android was chosen for its familiarity (Java) and open platform architecture. The overall architecture of the application includes searching, pairing and connecting to the Bluetooth module, receiving data via Bluetooth, processing the data using the seizure prediction algorithm, configuring the notification and patients settings and sending notifications. The Android OS has built-in interfaces to an RFCOMM class that assist with Bluetooth connections. While the OS handles the searching and pairing, the connection is maintained completely by the application. The mobile application implements a Bluetooth service that spawns a thread to handle setting up the connection in the background, along with another thread to handle the incoming data. The data are passed to another class via a Handler, which maintains a buffer and processes the data in chunks. When a seizure prediction biomarker occurs, a broadcast is sent to the notification system, which handles all of the notifications that were set up by the patient in the graphical UI (GUI) application. All the settings configured by the patient and the health provider are stored as private elements in Android's 'Shared Preferences' database. This was chosen because the variables can be accessed from any part of the mobile application, but can only be called from within the mobile application that created them. This ensures security and also allows the application to be extended for other purposes.

\section{Battery}

Portability and rechargeability are the primary concerns regarding the system's power supply. Considering this, the $5 \mathrm{~V} \mathrm{DC}-$ 1 A, myCharge Amp 4000 lithium polymer battery was chosen to support the system. The myCharge Amp interfaces with the system using a USB connection.

\section{Methods}

The architecture of the system enables the most efficient use of power usage and component flexibility throughout the system. An Arduino board is used to digitize the EEG signal, interface to an amplifier which would provide a high gain and low noise operation on the signal and provide an interface with a Bluetooth module to transmit the data. The bulk of data processing is performed 
on the Android device, where an application resides and can utilize the current power source of the smartphone. Therefore, the power consumption is minimized on the conditioning of the EEG signal, and data processing is managed as an application on an operating system environment. Through this architectural configuration, it is easy to update the software through application downloads and provide the capability for the smartphone to receive EEG data from a Bluetooth-enabled EEG data acquisition device.

There are 4 major components of our system: acquisition, processing, transmission and notification.

\section{Acquisition: Electrodes and Amplifier}

Four dry, active electrodes are used to detect the EEG signals. These small sensors are placed in various locations on the patient's head. A dry, passive electrode is placed on the patient's ear to serve as a reference. For convenience and a reliable connection, the electrodes are held by a headband (fig. 3).

The EEG signals are then fed into a 2-channel amplifier, the Olimex-SMT. This open-source amplifier is designed specifically to process EEG signals. The Olimex-SMT has 5 inputs and a single serial output. The inputs are described in the table 1 .

\section{Transmission: Bluetooth (SMT to Android)}

The data are then transmitted from the Olimex-SMT to the Android device for processing. To transmit the data via Bluetooth, RFCOMM sockets are established between the 2 devices. The serial port profile (SPP) HC-06 Bluetooth module is used to transmit USB serial data from the Olimex-SMT board to the Android device via the data pins. This establishes the Android device as the master and the Bluetooth module as the slave. Therefore, the Android application handles the searching, pairing and connecting to the Bluetooth module, and the module acts as a server that transmitted all of the data from it data pins over the sockets.

The app verifies the Bluetooth is ready, and then it spawns an Android activity to handle the searching and selecting via a device list. If the device has not been seen before, the user is prompted to input the required a secure connection (PIN code). Once a device has been selected from the list, the MAC address is passed to a Bluetooth service to handle the pairing and connection.

The Bluetooth service runs in the background and handles all of the pairing and connection tasks to set up the RFCOMM sockets. Once the sockets are established, the service uses an SPP profile to listen for and receive the data from the RFCOMM socket. The bytes received are then passed and stored into buffers for later processing. Once enough data have been collected, the buffers are passed to a Java class that handles processing the data using a seizure prediction algorithm.

\section{Processing: Data Processing}

As data are sent via Bluetooth and received by the Android device, it is placed in a buffer for processing. Once the buffer reaches a specific number of elements, a thread is spawned and the buffer is passed to the thread for processing. Once processing is complete, the buffer is flushed to allow new data to be acquired. Data structures were developed to help aid in the processing, including support for data sets that include complex numbers, filtering functions and multidimensional array operations. Based on the algorithm, the final output can be transformed into a binary output by

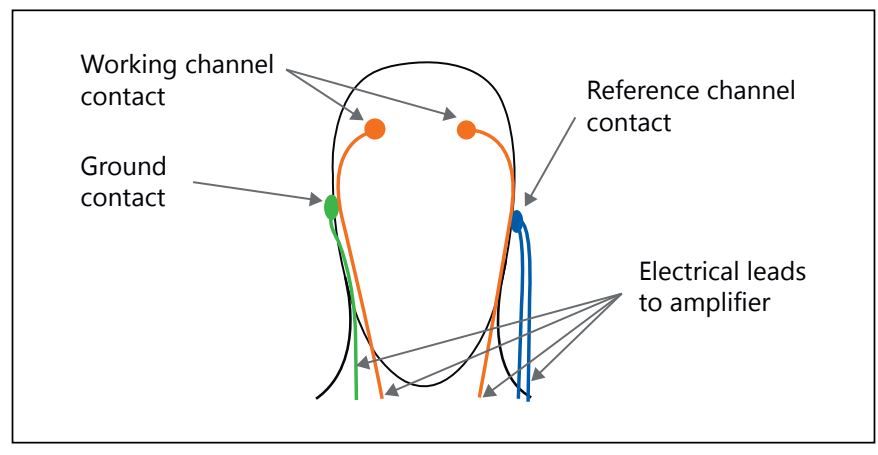

Fig. 3. Electrode configuration. EEG data acquisition (working) electrodes are applied to the forehead. Ground and reference electrodes are connected to earlobes. Electrodes lead to an amplifier for signals to be transmitted via Bluetooth module to smartphone for data processing.

Table 1. Electrode settings

\begin{tabular}{lll}
\hline Name & Description & Location \\
\hline $\mathrm{CH} 1+$ & $\begin{array}{l}\text { EEG data acquisition working } \\
\text { channel }\end{array}$ & Forehead \\
$\mathrm{CH} 2+$ & Reference & \\
$\mathrm{CH} 1-$ & Earlobe \\
\hline $\mathrm{DRL}$ & Ground & \\
\hline
\end{tabular}

using a threshold that is set to a specific value by the healthcare provider. The threshold value is established for each patient through an external algorithm. The value 'GlobalMax' is utilized to normalize the processed EEG to be between 0 and 1 . This value is patient specific. Once a seizure prediction biomarker is detected, the Android application constructs and commits the relevant notifications. The notification types and destinations are all configured by the user through the main UI of the Android application. The user can choose to send notifications via E-mail, SMS or use Android's notification system (i.e., pop-ups, notification bar, sounds, etc.).

\section{Notification: User Interface and Android Application Configuration}

Once a seizure prediction marker was detected, the application progresses through several conditional statements to determine how to send notifications. The user can select the type of notifications to use by activating or deactivating the radio buttons, which change flags in the background that affect the results of said conditional statements. The user can also personalize the notification recipients by using the appropriate buttons that are in the respective lists labeled 'SMS settings' and 'E-mail settings'. The 'Add' button spawns a pop-up window to allow the user to manually type in a contact or go to the phone's contacts to populate the lists. The user may remove recipients by using the appropriate 'Del' buttons for each contact in their respective list. Once the settings are final 
and the connection to the EEG acquisition device is initiated, the application will send the notifications based on these settings, that is, 'patient ID, date and time'.

\section{Results and Discussion}

\section{Individual Component Testing}

EEG Amplifier

To test the modular EEG SMT from Omilex, the device was plugged into a computer host via a USB cable to determine if the power LED indicator turned on. This ensured that the SMT could be powered with a $5 \mathrm{~V}$ USB power source.

\section{Bluetooth Module}

A breadboard, power supply and Arduino were used to test the Bluetooth module independently. The blinking LED on the Bluetooth module signified that power was being supplied. Next the module was interfaced with an Arduino Uno. This allowed for a computer to be connected and monitor the serial data from the module. The baud rate of the module was changed to match that of the EEG amplifier. Then the baud rate was verified using AT commands within the Arduino software.

\section{Battery}

Independent testing of the battery consisted of verifying the battery rating. This system used a myCharge Amp 4000, a $4000 \mathrm{mAh}$ Lithium-Polymer battery, rated for 5 $\mathrm{V}$ DC with an output current of $1 \mathrm{~A}$. The system requires significantly lower current. Therefore, the battery current was not a concern and the voltage was verified to be $5 \mathrm{~V}$ using a voltmeter.

\section{Mobile Phone}

The testing of the phone consisted of several parts: the Android application GUI, the notification system and the data processing. The main testing of the Android application involved ensuring that the GUI worked properly. All UI components functioned as intended, and the UI included the following design components: device connection, signal acquisition and notification settings. The GUI was tested by stepping through a workflow and ensuring that the functionality of every button, checkbox, list view and image were working properly. The notification system involved taking user-designated input to form local notifications (i.e., popups) and sending notifications to others via SMS or E-mail. The system was tested by traversing the GUI and selecting the desired

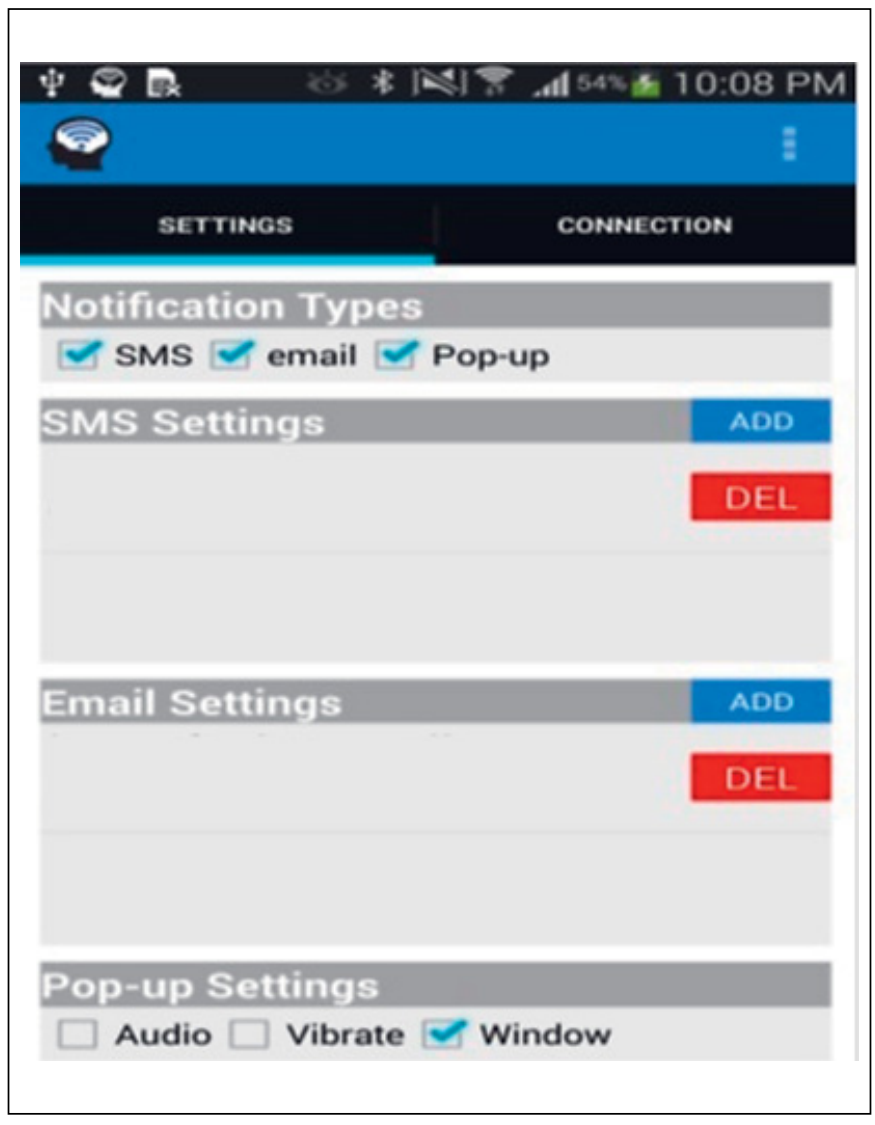

Fig. 4. Notification settings. The notification system is configurable using the GUI of the Android application. Upon successful detection of a seizure prediction biomarker, the notification system can send a notification via SMS, E-mail or an Android popup notification.

notification settings, then creating pseudo-seizure events to trigger the notifications. The system passed this test when such seizure events triggered the correct notifications (fig. 4).

\section{Multiple Component Testing}

\section{Electrode + Amplifier}

The simplest way to verify that the electrodes were acquiring EEG signals was to use the electrodes in conjunction with the amplifier and then monitor the signals using the BrainBay software. BrainBay is a bio-neuro feedback application designed to work with the OpenEEG hardware, ModularEEG and MonolithEEG. BrainBay is part of the OpenEEG project and provides graphical feedback. In our design, we used BrainBay for bioelectrical signal processing and graphing. The electrodes were connected to the amplifier and the amplifier into the USB port of a computer containing the BrainBay software. After the 
electrodes were placed on a subject's head, the EEG signals were viewed on the computer. To verify that the signals were not noise, the person could blink and the EEG signals on the computer spiked.

\section{Bluetooth + Phone}

After the Bluetooth module was successfully powered by the battery, the orange LED on the module blinked. Then, the user went to connection tab and selected search for device.Then, theBluetoothmodulenamedSeizMonitorHealth device was selected. After the popup disappeared, 'connected to SeizMonitorHealth device' appeared on the phone, and the diagram displayed a valid connection (fig. 5). This means that the devices were paired. At this point, the orange LED stopped blinking and glowed steadily.

\section{Phone + Algorithm}

Using the test data set, the data was processed on the mobile device and the results were passed to the notification system. When a seizure prediction biomarker was encountered, the notification system responded with the appropriate notifications based on the user-specified settings (i.e., SMS, E-mail, popup).

\section{Electrode + Amplifier + Bluetooth}

Once the electrode + amplifier system was confirmed to be working, the Bluetooth module was added and verified using a computer. The system was powered by the computer during this testing. Electrodes were placed on a subject via the cap, and the electrodes were connected to the appropriate channels on the amplifier. The amplifier was connected to the Bluetooth module and began sending data. The transmitted data was verified using a Bluetooth-enabled device.

\section{System Testing}

Electrode + Amplifier + Bluetooth + Phone + Battery

To verify the functionality of the complete system, the amplifier and Bluetooth was initially powered by the battery. Also, the phone was paired with the Bluetooth model. The app was in the Healthcare Config window. After the electrodes were appropriately placed on a subject, the channel 1 and 2 graphs updated in real time the EEG signals. A similar test to the electrode + amplifier test was conducted in order to confirm that the appropriate signals were being acquired. Data acquisition is performed via the EEG SMT Board, which gathers EEG data from active electrodes (fig. 6). The wireless communication is implemented using Bluetooth with baud rate set to 57,600 , which transmits data sampled at $256 \mathrm{~Hz}$.

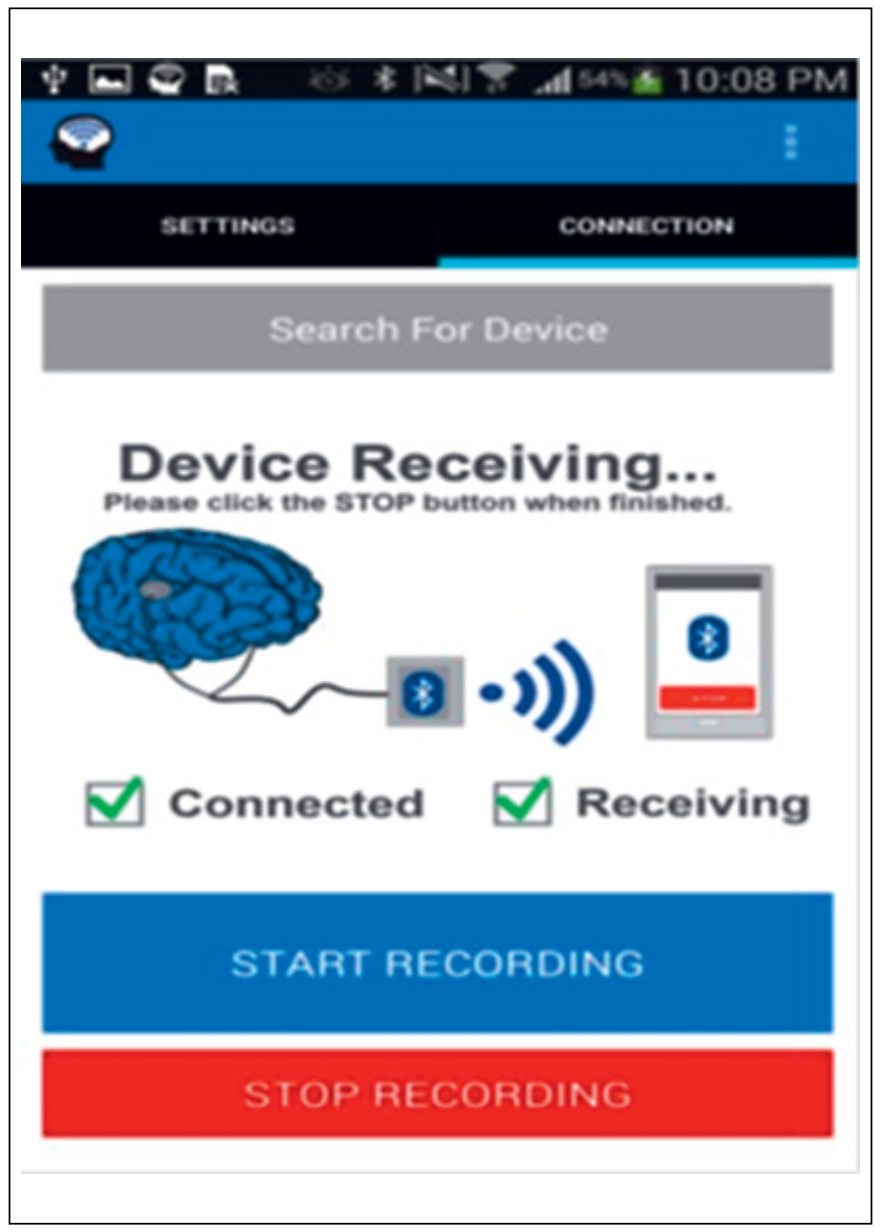

Fig. 5. Data pairing between amplifier and smartphone. The amplifier sends EEG data via Bluetooth module to smartphone. The Bluetooth module searches for the available smartphone to process data.

\section{Power Consumption}

The power consumption of the system was analyzed in 2 parts: the power consumption of the external battery as well as the effect of the app on the phone battery. To calculate the power consumed by the electrodes, amplifier and Bluetooth module on the external battery, the voltage from the battery was measured using a voltmeter and the current from the battery to the components. In order to calculate the maximum power consumption, the measurements were taken when the system was acquiring and transmitting data. It is difficult to quantitatively analyze the effect of the app on the phone battery. However, a general idea was gained by contrasting the battery life when the app was running/not-running. In addition, there are several diagnostic phone applications that estimate the power drain caused by aparticular application, 


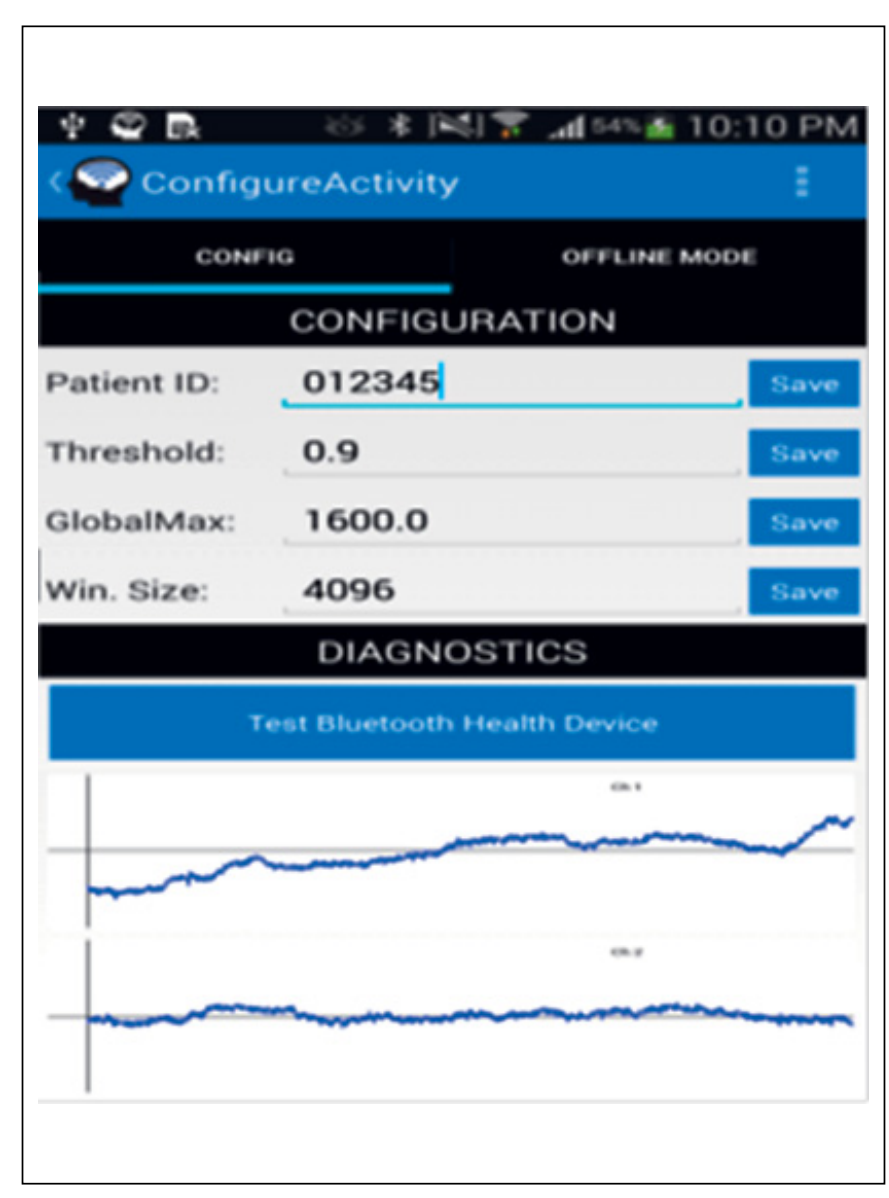

Fig. 6. Patient configuration. The patient ID identifies specific transmitted data stream. The value 'threshold' separates seizure states from non-seizure states by setting a target for processed EEG data that rises above a patient-specific value, from $(0,1)$. 'GlobalMax' normalizes processed data from ' 0 ' to ' 1 ' based on the data segment (window size), so that seizure states can be processed and detected.

for example, Battery Stats Plus. This information was also considered. The output voltage was measured to be $\mathrm{V}=$ $5.049 \mathrm{~V}$ with $\mathrm{I}=99.077 \mathrm{~mA}$. Therefore,

$$
\mathrm{P}=\mathrm{IV}=5.049 \mathrm{~V} \times 99.077 \mathrm{~mA}=500.24 \mathrm{~mW}
$$

\section{Lifetime - Online Testing}

The system lifetime was evaluated by monitoring how long the system could continuously acquire, transmit and process the EEG data. Data were collected and transmitted to the phone to be processed. The lifetime of the system was observed to be $12 \mathrm{~h}$, where all components of the system were operating under maximum load. Also, the system is capable of acquiring and processing real time data with less than a seconds delay.

Ambulatory Seizure Monitoring: From Concept to Prototype Device
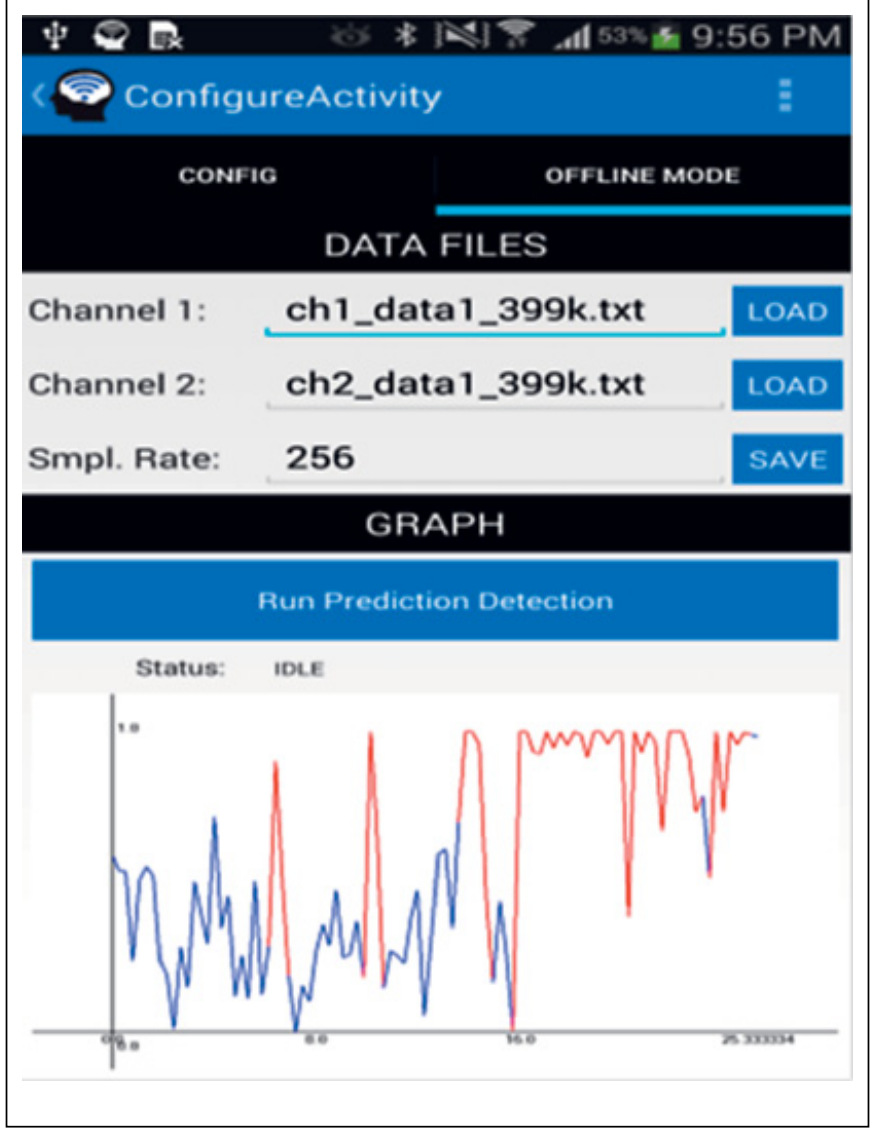

Fig. 7. Offline mode. Offline mode enables device testing based on previously recorded EEG seizure data. This screen can be used for testing additional EEG data acquisition systems, where the sampling rate of the collected data is entered based in the acquisition system. The 'run prediction detection' display features processed EEG seizure data, where lines colored in red demonstrate pre-seizure states (predicted) and post-seizure states (detected).

\section{Offline Testing}

Offline testing was conducted using an EEG data set. The data were loaded into the mobile phone application, processed and then the notifications were sent.

Seizure detection utilizes the seizure prediction and detection algorithm described previously and will perform as shown in figure 7 .

\section{Experimental Results of Seizure Monitoring: \\ Prediction and Detection Markers}

A scenario where the seizure monitoring system may be considered involves patients with intractable types of seizures who are candidates for vagus nerve stimulator (VNS) implants. A physician would want to monitor the patient before performing the surgery in order to

Ann Neurosci 2016;23:100-111 
Table 2. Number of seizures detected pre- and post-implant per frequency band filter range and window size

\begin{tabular}{|c|c|c|c|c|c|c|c|c|c|c|c|c|c|}
\hline \multirow{2}{*}{\multicolumn{2}{|c|}{$\frac{\text { Filter band, } \mathrm{Hz}}{\text { Window size (samples) }}$}} & \multicolumn{2}{|l|}{$1-12$} & \multicolumn{2}{|l|}{$6-12$} & \multicolumn{2}{|c|}{$12-20$} & \multicolumn{2}{|c|}{$20-30$} & \multicolumn{2}{|c|}{$30-40$} & \multicolumn{2}{|l|}{$40-50$} \\
\hline & & \multirow[t]{2}{*}{1,000} & \multirow[t]{2}{*}{3,000} & \multirow[t]{2}{*}{1,000} & \multirow[t]{2}{*}{3,000} & \multirow{2}{*}{\multicolumn{2}{|c|}{$1,0003,000$}} & \multirow{2}{*}{\multicolumn{2}{|c|}{$1,0003,000$}} & \multirow[t]{2}{*}{1,000} & \multirow[t]{2}{*}{3,000} & \multirow{2}{*}{\multicolumn{2}{|c|}{$1,0003,000$}} \\
\hline & Implant* & & & & & & & & & & & & \\
\hline \multirow[t]{2}{*}{1} & Pre (1) & 1 & 1 & 1 & 1 & & & & & 1 & 1 & 1 & 1 \\
\hline & Post (5) & 5 & 5 & 5 & 5 & 5 & 5 & 5 & 5 & 5 & 5 & 5 & 5 \\
\hline \multirow[t]{2}{*}{2} & Pre (4) & 2 & 1 & 3 & & & & & & & & 3 & 1 \\
\hline & Post (5) & 4 & 2 & 5 & 2 & & & & & & & 2 & 1 \\
\hline \multirow[t]{2}{*}{3} & Pre (4) & 1 & & 2 & & & & & & 1 & & 2 & \\
\hline & Post (5) & 4 & 3 & 1 & 4 & & & & & & & 2 & 4 \\
\hline
\end{tabular}

* Number of actual seizures is given in parentheses.

Table 3. Number of seizures predicted pre- and post-implant per frequency band filter range and window size

\begin{tabular}{|c|c|c|c|c|c|c|c|c|c|c|c|c|}
\hline \multirow{2}{*}{\multicolumn{2}{|c|}{$\frac{\text { Filter band, } \mathrm{Hz}}{\text { Window size (samples) }}$}} & \multicolumn{2}{|l|}{$1-12$} & \multicolumn{2}{|l|}{$6-12$} & \multirow{3}{*}{$\frac{12-20}{1,0003,000}$} & \multicolumn{2}{|l|}{$20-30$} & \multicolumn{2}{|c|}{$30-40$} & \multicolumn{2}{|l|}{$40-50$} \\
\hline & & \multirow[t]{2}{*}{1,000} & \multirow[t]{2}{*}{3,000} & \multirow[t]{2}{*}{1,000} & \multirow[t]{2}{*}{3,000} & & \multirow{2}{*}{\multicolumn{2}{|c|}{$1,000 \quad 3,000$}} & \multirow[t]{2}{*}{1,000} & \multirow[t]{2}{*}{3,000} & \multirow[t]{2}{*}{1,000} & \multirow[t]{2}{*}{3,000} \\
\hline Patient & Implant* & & & & & & & & & & & \\
\hline \multirow[t]{2}{*}{1} & Pre (1) & 1 & 1 & 1 & 1 & & & & 1 & 1 & 1 & 1 \\
\hline & Post (5) & 2 & & 4 & 1 & & & & & & 3 & 1 \\
\hline \multirow[t]{2}{*}{2} & Pre (4) & 2 & 1 & & & & & & & & 2 & 1 \\
\hline & Post (5) & 4 & 2 & 5 & 2 & & & & & & 2 & 1 \\
\hline \multirow[t]{2}{*}{3} & Pre (4) & 1 & & 2 & & & & & & & 2 & \\
\hline & Post (5) & 4 & 3 & 1 & 2 & & & & & & 2 & 4 \\
\hline
\end{tabular}

* Number of actual seizures is given in parentheses.

gather frequency and intensity of the seizure state. Additionally, seizure monitoring involves gathering attributes of the neural signals, such as band pass frequency selection and window size for data processing of incoming signal.

EEG data have been used to test the algorithm for this device from patients with medically intractable epilepsy, who were candidates for the VNS surgical treatment. Features of normal/abnormal brain activity have been monitored for 60-90 min. Action potentials are captured at a sampling rate of 250 points/second. Testing of the seizure monitoring algorithm features EEG recordings that were collected from 3 patients during pre- and post-VNS implant recording. The length of ictal behavior ranged from 8 to $12 \mathrm{~s}$. Tables 2 and 3 displays the number of seizures per patients, seizures detected and seizures predicted by our algorithm. EEG filtering was accomplished using a
Remez filter in brain frequency ranges delta-theta-alpha (1-12 Hz), alpha $(6-12 \mathrm{~Hz})$, beta $(13-30 \mathrm{~Hz})$, gamma $(30-40 \mathrm{~Hz})$ and upper-gamma $(40-50 \mathrm{~Hz})$.

The experimental findings illustrate that there may be a triggering mechanism through the PLV method that enables seizure prediction.

This analysis features the number of seizures predicted and found using the PLV methodology. Seizure prediction and detection sensitivity is displayed in the following graphs in figure $8 \mathrm{a}-\mathrm{f}$. Sensitivity calculations were processed per patient. Sensitivity calculations use the total number of pre- and post-implant seizures per patient that were identified by the clinician who provided the EEG data set for this research. This value is used to calculate the number of true positives.

The highest seizure detection sensitivity for patients 1 , 2 and 3 was from 100,75 and $80 \%$, respectively, depending 


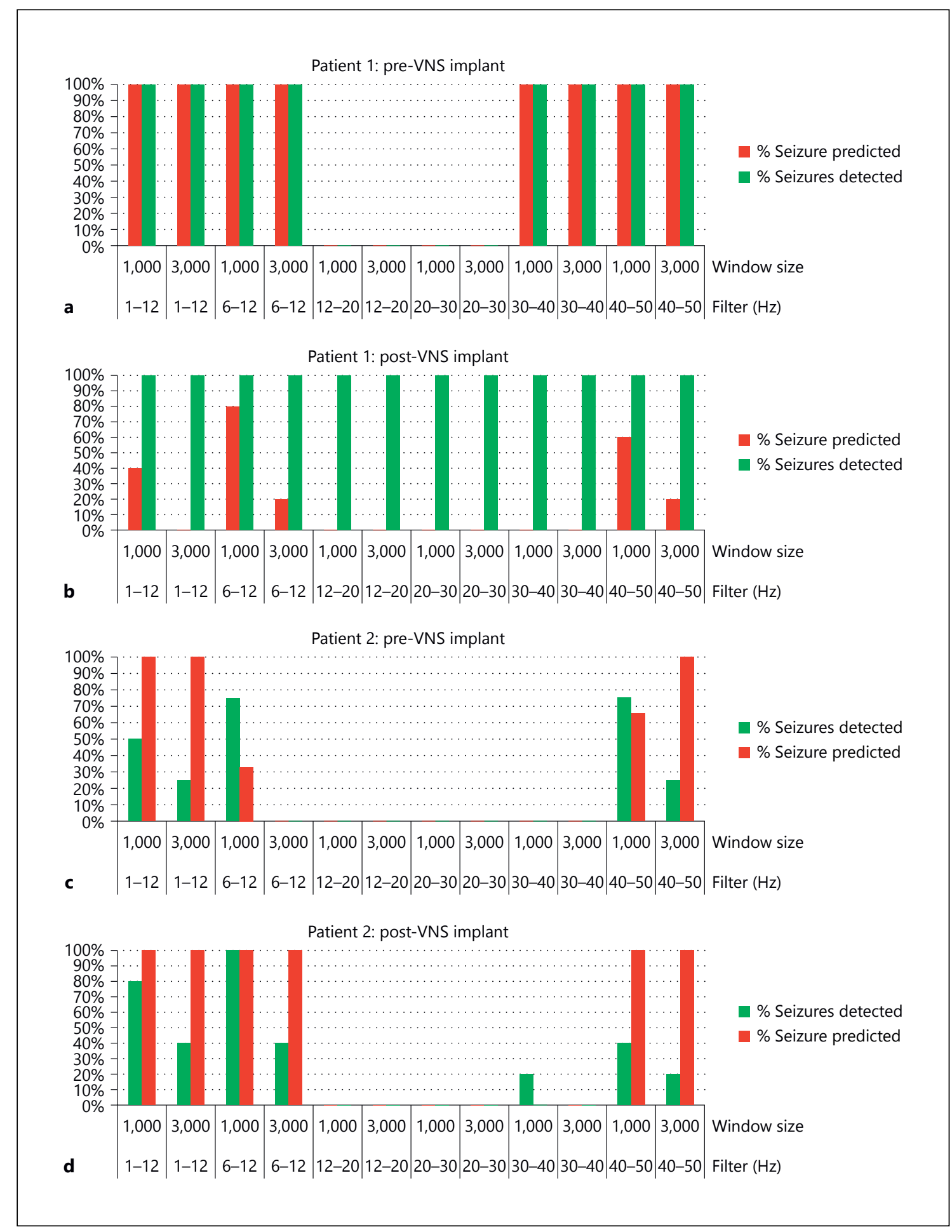

Fig. 8. Seizure detection and prediction sensitivity graphs. Patient 1: pre- and post-VNS implant detection and sensitivity displayed in (a) and (b), patient 2 values in pre (c) and post (d) VNS implants and patient 3 values in pre (e) and post (f) VNS implants.

(For figure $8 e$ and $f$ see next page.) 


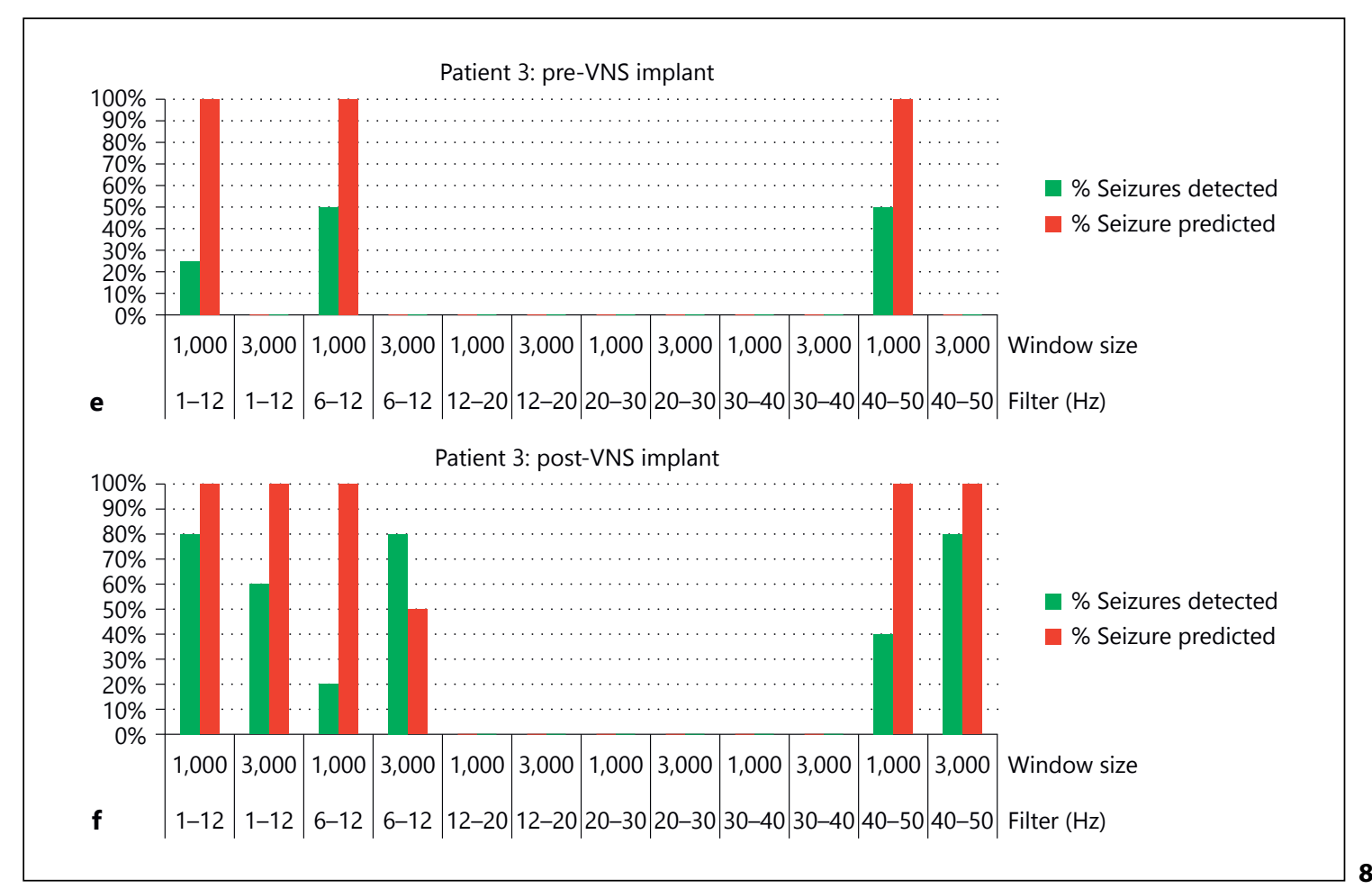

on frequency band selection. The highest seizure prediction sensitivity for patients 1,2 and 3 was 80,100 and $100 \%$, respectively. The bandwidth $6-12 \mathrm{~Hz}$ (alpha) has the most occurrences of seizure prediction and detection markers across all the pre- and post-implant EEG recordings.

The system architecture and the algorithm facilitate an EEG monitoring capability that provides a better understanding of the patient's seizure condition and enables an enhanced approach to patient monitoring. Ultimately, the patient will be provided the best intervention based on the analysis of this device.

\section{Conclusion}

According to the Centers of Disease Control and Prevention, roughly 2.3 million adults and 467,711 children in the United States have epilepsy. Nearly, 150,000 Americans develop the condition every year [12]. People with epilepsy live in constant fear of an impending seizure. For extreme cases, they are unable to drive or do many of the daily functions most people take for granted and therefore are limited in the daily activities. When a patient with epilepsy has a seizure, they may stay in a hospital bed while EEG tests are being done in an attempt to diagnose the cause and severity of the sei- zures. This device addresses the need for long-term monitoring of the patient's seizure condition in order to provide the clinician a better understanding of the seizure's duration and frequency, and ultimately provide the best remedy for the patient.

\section{Acknowledgments}

The data for this study is provided by Dr. James W. Wheless, MD, Professor and Chief of Pediatric Neurology and LeBonheur Chair in Pediatric Neurology, University of Tennessee Health Science Center. Experiments were conducted in accordance to The University of Memphis IRB regulations, IRB reference protocol: E06-80.

\section{Author Contributions}

M.H.M. and K.A.S. developed concept; M.H.M. developed algorithm, architecture and developed manuscript; K.A.S. and J.D.B. proofread manuscript; J.D.B. provided hardware analysis; M.T. developed initial version of manuscript; M.T., K.M.S., B.M.M. and L.B.H. developed prototype.

\section{Disclosure Statement}

The authors declare no conflict of interest. 


\section{References}

1 Chi YM, Wang YT, Wang Y, Maier C, Jung TP, Cauwenberghs G: Dry and noncontact EEG sensors for mobile brain-computer interfaces. IEEE Trans Neural Syst Rehabil Eng 2012;20:228-235.

2 Matthews R, Turner PJ, McDonald NJ, Ermolaev K, Manus T, Shelby RA, Steindorf M: Real time workload classification from an ambulatory wireless EEG system using hybrid EEG electrodes. Conf Proc IEEE Eng Med Biol Soc 2008;2008:5871-5875.

3 Lin R, Lee RG, Tseng CL, Wu YF, Jiang JA: Design and implementation of wireless multichannel EEG recording system and study of EEG clustering method. Biomed Eng Appl Basis Comm 2006;18:276-283.

4 Lin CT, Ko LW, Chiou JC, Duann JR, Huang RS, Liang SF, Chiu TW, Jung TP: Noninvasive neural prostheses using mobile and wireless EEG. Proc IEEE 2008;96:1167-1183.

5 Lin CT, Lin BS, Lin FC, Chang CJ: Brain computer interface-based smart living environmental auto-adjustment control system in UPnP home networking. IEEE Syst J 2014;8:363-370.

6 Sackellares JC, Iasemidis LD, Shiau DS: Detection of preictal transition in scalp EEG. Epilepsia 1999;40:176.

7 Myers MH, Kozma R: Simulations of Seizure Detection for Vagus Nerve Stimulation Patients. San Diego, Neuroscience SfN 40th Annual Meeting, 2010.

8 Myers MH, Kozma R: Modeling Brain Electrical Activity Involving Vagus Nerve Stimulation. St. Louis, Intelligent Engineering Systems Through Artificial Neural Networks, 2007, vol 17, pp 3-8.
9 Majumdar K, Myers MH: Amplitude suppression and chaos control in epileptic EEG signals. Comput Math Methods Med 2006;7: 53-66.

10 Myers MH, Padmanabha A, Hossain G, de Jongh Curry AL, Blaha CD: Seizure prediction and detection via phase and amplitude lock values. Front Hum Neurosci 2016;10:80.

11 Worrell GA, et al: High-frequency oscillations and seizure generation in neocortical epilepsy. Brain 2004;127(pt 7):1496-1506.

12 Jacobs J, et al: High-frequency electroencephalographic oscillations correlate with outcome of epilepsy surgery. Ann Neurol 2010; 67:209-220.

13 Center for Disease Control and Prevention. http://www.cdc.gov/epilepsy/basics/faqs. htm. 